\title{
Neural Networks applied to wireless communications
}

\author{
Georgina Stegmayer ${ }^{1}$ and Omar Chiotti ${ }^{2}$ \\ 1 C.I.D.I.S.I., Universidad Tecnológica Nacional, Lavaise 610, 3000 Santa Fe, \\ Argentina. e-mail: gstegmay@frsf .utn.edu.ar \\ 2 INGAR-CONICET, Avellaneda 3654, 3000 Santa Fe, Argentina. e-mail: \\ chiotti@ceride.gov.ar
}

\begin{abstract}
This paper presents a time-delayed neural network (TDNN) model that has the capability of learning and predicting the dynamic behavior of nonlinear elements that compose a wireless communication system. This model could help speeding up system deployment by reducing modeling time. This paper presents results of effective application of the TDNN model to an amplifier, part of a wireless transmitter.
\end{abstract}

\section{Introduction}

In new generation wireless communications - i.e. third generation (3G) standards such as WCDMA (Wideband Code Multiple Division Access) and UMTS (Universal Mobile Telecommunications System) towards which most of the current cellular networks will migrate - system component modeling has become a critical task inside the system design cycle, due to modern digital modulation schemes [1].

New standards may introduce changes in the behavior of the devices that are part of the system (e.g. mobile phones and their internal components) mainly due to the modulation schemes they use, generating nonlinearities in the behavior and memory effects (when an output signal depends on past values of an input signal). Memory effects in the time-domain cause the output of an electronic device to deviate from a linear output when the signal changes, resulting in the deterioration of the whole system performance since the device begins behaving nonlinearly. In this work we are interested in modeling the nonlinear behavior that an amplifier can have inside a wireless transmission.

Amplifiers are a major building block of modern RF digital wireless transmitters (i.e. cellular phones). Figure 1 shows a simplified block diagram of what a ccllular phone communication would be. The voice coming from the phone speaker (analog signal) has to be digitalized to be transmitted through the wireless network, and this is the task of an Analog/Digital converter. The digitalized voice then has to be compressed to reduce bit rate and bandwidth. It is also codified, to format the data so the receiver can detect and minimize errors by doing the reverse operation. After that, a modulator adds the carrier signal to

Please use the following format when citing this chapter:

Stegmayer, G., Chiotti, O., 2006, in IFIP International Federation for Information Processing, Volume 217, Artificial Intelligence in Theory and Practice, ed. M. Bramer, (Bosten: Springer), pp. 129-138. 
the data signal. The signal has to reach an antenna from the cellular phone with enough strength to guarantee the communication. But the signal suffers from attenuation and needs amplification before that. Therefore, the final element of the chain is a power amplifier (PA) which amplifies the signal before it travels to the nearer antenna and to the receiver side of the communications chain.

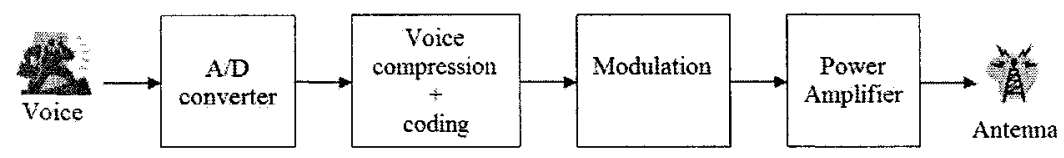

Fig. 1. Simplified block diagram of a digital wireless transmitter.

An amplifier works by increasing the magnitude of an applied signal. Amplifiers can be divided into two big groups: linear amplifiers, which produce an output signal directly proportional to the input signal, and power amplifiers which have the same function as the first ones, but their objective is to obtain maximum output power. A PA can work in different "classes": in Class A if it arrives at the limit of the linearity; and class $B$ when it works in nonlinear regime. Moreover, in wireless communications, the transmitter itself introduces nonlinearities when operating near maximum output power [2].

Nonlinear behavior modeling has been object of increasing interest in the last years [3][4] since classical techniques that were traditionally applied for modeling are not suitable anymore. That is why new techniques and methodologies have been recently proposed, as for example neural network (NN) based modeling applied to PA modeling[5].

Neural networks, as a measurement-based technique, may provide a computationally efficient way to relate inputs and outputs, without the computational complexity of full circuit simulation or physics level knowledge [6], therefore significantly speeding up the analysis process. No knowledge of the internal structure is required and the modeling information is completely included in the device external response.

Although the NN approach has been largely exploited for static simulation, their application to dynamic systems modeling is a rather new research field. In this paper we present a new NN model for modeling nonlinear elements that belong ro a communications chain, using a network which takes into account device nonlinearity and memory effects. In particular, this paper presents the results of the proposed model to nonlinear PA modeling.

The organization of the paper is the following: in the next Section, NN-based modeling of electronic components is presented. Section 3 explains the neural network model presented in this paper and shows its architecture and parameters. In Section 4, measurcments and validation results are shown. Finally, the conclusions are reported in Section 5. 


\section{Neural network-based modeling}

Neural network-based models are nowadays seen as a potential alternative for modeling electronics elements having medium-to-strong memory effects along with high-order nonlinearity. NNs are preferred over traditional methods (i.e. equivalent-circuit, empirical models) because of their speed in implementation and accuracy. A NN model can be used during the stage of system design for a rapid evaluation of its performance and main characteristics. The model can be directly trained with measurements extracted from the real system, speeding up the design cycle. NN models can be more detailed and rapid than traditional equivalent-circuit models, more exact and flexible than empirical models, and easier to develop when a new technology is introduced. By profiting from their potential to learn a device behavior based on simulated or measured records of its input and output signals, they were used in nonlinear modeling and design of many microwave circuits and systems [7].

The increasing number of electronic devices models proposed using NNs that have appeared in the last years [8][13][10] shows their importance and interest. Many topologies of NNs are reported in the literature for modeling different types of circuits and systems, with different kinds of linear and nonlinear behavior [11]. However, until very recently, NNs for modeling were applied almost exclusively to instantaneous behavior of the input variables alone. Although this approach has been largely exploited for static simulation, their application to dynamic system is a rather new research field. Recently have appeared NNbased models taking into account the dynamic phenomena in RF microwave devices [12].

For representation of a system which has a nonlinear behavior and is dynamic, intending by dynamic not only that the device characteristic varies over time but also that it depends on past values of its controlling input variables, not any $\mathrm{NN}$ topology can be used. A neural model which includes time-dependence into the network architecture is the time-delayed neural network (TDNN), a special type of the well-known multilayer-perceptron (MLP). TDNNs have been successfully applied for solving the temporal processing problems in speech recognition, system identification, control and signal modeling and processing [13]. They are suited for dynamic systems representation because the continuous time system derivatives are approximated inside the model by discrete time-delays of the model variables.

A TDNN is based on the feedforward MLP neural network with the addition of tapped delay lines $\left(Z^{-1}\right)$ which generate delayed samples of the input variables. They are used to add the history of the input signals to the model, needed for memory effects modeling. The TDNN entries include not only the current value of the input signal, but also its previous values, as illustrates figure 2. The memory depth $M$ of the element or system analyzed is reflected on the length of the taps. The strategy followed to set the system memory is dictated by the bandwidth accuracy required. 


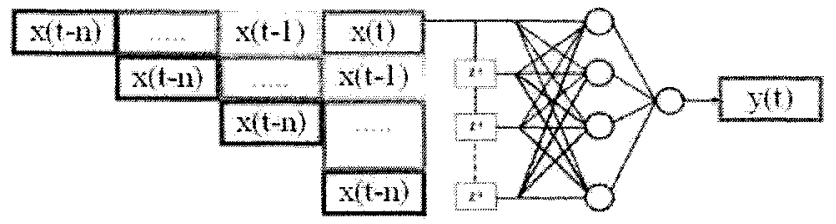

Fig. 2. Time-Delayed neural network (TDNN) model and its corresponding input data.

In this paper we propose the application of a TDNN model for modeling nonlinear and dynamic behavior of devices or elements, parts of a communication system. The model proposed is explained in detail in the next Section.

\section{TDNN model}

The proposed model has the classical three layers topology for universal approximation in a MLP: the input variable and its delayed samples, the nonlinear hidden layer and a linear combination of the hidden neurons outputs at the output neuron. The architecture of the TDNN is shown in figure 3.

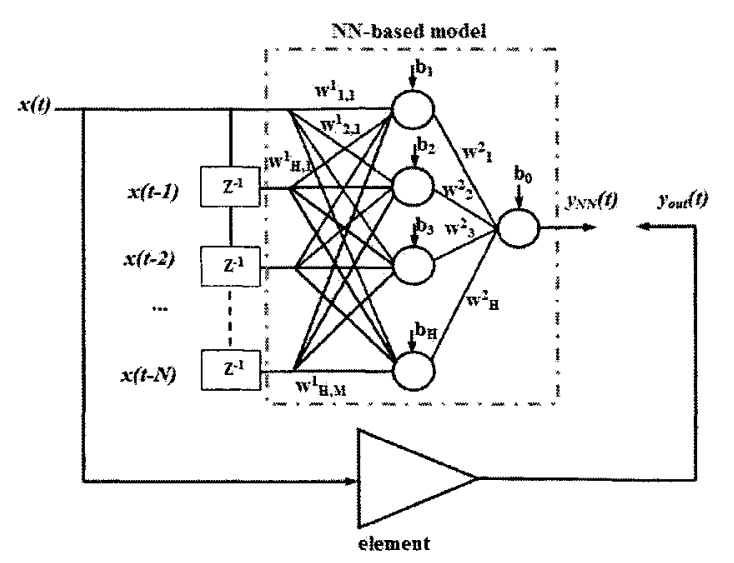

Fig. 3. Time-Delayed neural network (TDNN) to model a communications chain component.

The input layer has as inputs the samples of the independent variable, together with its time-delayed values. The model here presented shows a one-input variable dependence $(x)$ of one output variable $(y)$, but the model can be easily extended to more input/output variables. The variable can have a delay tap between 0 and $N$, then the total number of input neurons is $\mathrm{M}(M=N+1)$. 
In the hidden layer, the number $h$ of hidden neurons varies between 1 and $H$. The hidden units have a nonlinear activation function. In general, the hyperbolic tangent (tanh) will be used because this function is usually chosen in the electronics field for nonlinear behavior. In our model we have used $\mathrm{H}=$ 10 , but if necessary, the number of neurons in the hidden layer can be changed to improve the network accuracy. The hidden neurons receive the sum of the weighted inputs plus a corresponding bias value for each neuron $b_{h}$. All the neurons have bias values. This fact gives more degrees of freedom to the learning algorithm and therefore more parameters can be optimized, apart from weights, to better represent a nonlinear system.

The weights between layers are described with the usual perceptron notation, where the sub-indexes indicate the origin and the destination of the connection weight, e.g. $w_{j, i}$ means that the weight $w$ relates the destination neuron $j$ with the origin neuron $i$. However, a modification in the notation was introduced, adding a super-index to the weights, to more easily identify to which layer they belong. Therefore, $w^{1}$ means that the weight $w$ belongs to the connection between the inputs and the first hidden layer, and $w^{2}$ means that the weight $w$ belongs to the connection between the first hidden layer and the second one (in our particular case, the second hidden layer happens to be the output layer). In this second group of connections weights, the sub-index which indicates the destination neuron has been eliminated because there is only one possible destination neuron (the output).

This unique output neuron has a linear activation function which acts as a normalization neuron (this is usual choice in MLP models). Therefore, the output of the proposed TDNN model is calculated as the sum of the weighted outputs of the hidden neurons plus the corresponding output neuron bias $\left(b_{0}\right)$, yielding equation 1 .

$$
y_{N N}(t)=b_{0}+\left[\sum_{h=1}^{H} w_{h}^{2} \tanh \left(b_{h}+\sum_{i=0}^{N} w_{h, i+1}^{1} x(t-i)\right)\right]
$$

Network initialization is an important issue for training the TDNN with the back-propagation algorithm, in particular in what respects speed of execution. In this work the initial weights and biases of the model are calculated using the Nguyen-Widrow initial conditions [14] which allow reducing training time, instead of a purely random initialization.

Once the TDNN model has been defined, it is trained with time-domain measurements of the element output variable under study $\left(y_{o u t}(t)\right)$, which is expressed in terms of its discrete samples. To improve network accuracy and speed up learning, the inputs are normalized to the domain of the hidden neurons nonlinear activation functions (i.e for the hyperbolic tangent tanh, the interval is $[-1 ;+1])$. The formula used for normalization is shown in equation 2.

$$
x^{\text {norm }}=\frac{2(x-\min \{x\})}{(\max \{x\}-\min \{x\})}-1
$$


During training, network parameters are optimized using a backpropagation algorithm such as the Levenberg-Marquardt [15], chosen due to its good performance and speed in execution. To evaluate the TDNN learning accuracy, the mean square error (mse) is calculated at each iteration $k$ of the algorithm, using equation 3, where $P$ is the number of input/output pairs in the training set, $y_{\text {out }}$ is the output target and $y_{N N}$ is the NN output.

$$
m s e=\frac{1}{P} \sum_{k=1}^{P} E(k)^{2}=\frac{1}{P} \sum_{k=1}^{P}\left(y_{\text {out }}(k)-y_{N N}(k)\right)^{2}
$$

The good generality property of a NN models says that it must perform well on a new dataset distinct from the one used for training. Even a excessive number of epochs or iterations on the learning phase could make performance to decrease, causing the over-fitting phenomena. That is why, to avoid it, the total amount of data available from measurements is divided into training and validation subsets, all equally spaced. We have used the "early-stopping" technique [16], where if there is a succession of training epoch in which accuracy improves only for the training data and not for the validation data, over-fitting has occurred and the learning is terminated. The obtained results are shown in the next section.

\section{Measurements and validation results}

For training the neural model, a dedicated test-set for accurate PA characterization has been used. It provides static and pulsed DC characterization, scattering parameter measurements, real-time load/source-pull at fundamental and harmonic frequencies, and gate and drain time-domain RF waveforms. The measurements are carried out with a Microwave Transition Analyzer and a large-signal Vector Network Analyzer.

Complete characterization was performed for different input power levels and different classes of operation at $1 \mathrm{GHz}$ on a 2 ns window, as shows figure 4 . Class A is biased at $50 \%$ IDSS, and class B with $I D S=0$. A $1 \mathrm{~mm}$ total gate periphery GaN HEMT based on $\mathrm{SiC}$ with $\mathrm{IDSS}=700 \mathrm{~mA}$, has been measured at $1 \mathrm{GHz}$. The power sweep ranged from -21 to $+27 \mathrm{dBm}$. Only the first 4 harmonics are taken into account.

The basic idea to characterize the nonlinear models for IDS is to collect input-output data with different tuned-load terminations, mapping the widest region in the $I-V$ characteristic. Time-domain data have been collected only for load-pull characterization results in this case, that is three tuned-loads, $50 \mathrm{ohm}$, the best output power (Pout) and the best output efficiency (PAE). The dynamic load curves corresponding to the selected loads are rather close in class A operation, whereas they are fairly open in class B operation. This suggested to use the all three selected loads from class B characterization, and only $50 \mathrm{ohm}$ load data from class $A$. The other characterization data will be 

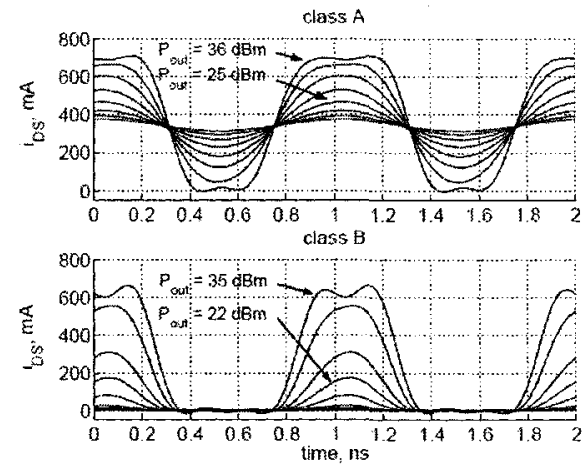

Fig. 4. Time-domain waveforms at $1 \mathrm{GHz}$ at increasing power for class A (top), and $\mathrm{B}$ (bottom) at $50 \mathrm{Ohm}$ load used for training.

used for model validation. In principle, however, this method does not need full load-pull characterization, but only to map gate and drain nonlinear models for different generic tuned-loads, in order to concern the widest region in the I-V characteristic. Input data vectors of VGS and VDS for each load and power level have been first copied and delayed as many times, to represent the network inputs, as necessary to account for memory, and then joined together to train the TDNN model with all the working classes, the selected load terminations and the power levels, simultaneously. This is shown in figure 5 .

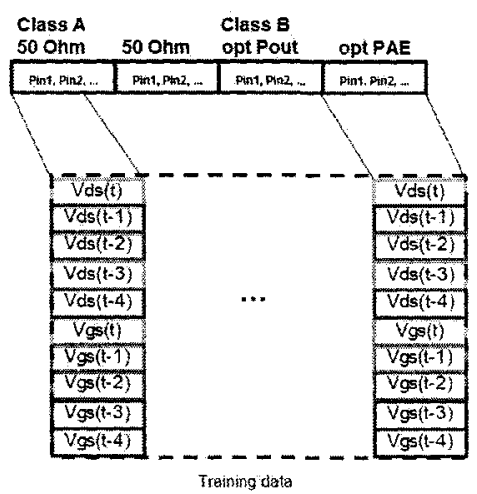

Fig. 5. TDNN model training data organization.

We report here some results for class $\mathrm{A}$ and $\mathrm{B}$, at $\mathrm{VDS}=30 \mathrm{~V}$. The validation test includes the best Pout load, that has not been used for model training, and some intermediate points for input power levels not included in the training set. Results obtained show a rather good agreement between experimental and 
modelled data, in fact the relative mean square error is lower than 1e-04 (figure $6)$.

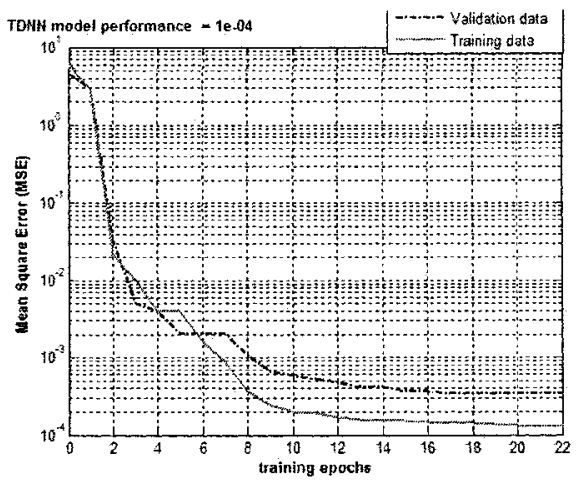

Fig. 6. TDNN model performance.

Figure 7 reports the output PA time-domain waveforms at increasing output power at $50 \mathrm{Ohm}$ load: the upper plots are relative to a class $\mathrm{A}$ condition, while the bottom ones refer to class B operations. The left figures report the measurements, while the right ones show the TDNN simulation result. Figure 8 shows the comparison between the training (left) and validation (right) measurement data used for class $\mathrm{A}$ operation and the TDNN model response.
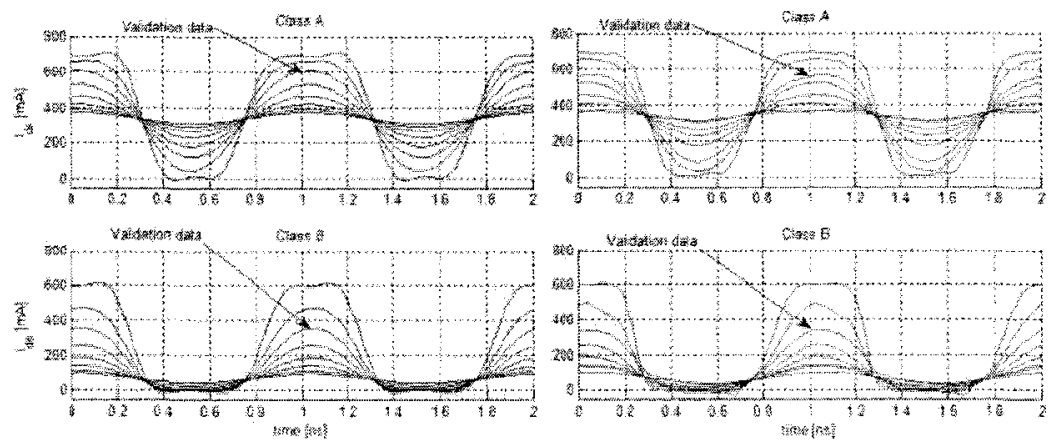

Fig. 7. Time-domain waveforms at $1 \mathrm{GHz}$ at increasing power for class $\mathrm{A}$ (top), and $\mathrm{B}$ (bottom). Measurements (left), TDNN output (right). The pointed arrow waveform refers to a validation data subset.

From the comparison between measurements and model output, the waveforms good agreement in general. Also for the power levels excluded from the training data, used only for model validation. This is a key result to prove the 
model predictive capabilities. The model is also capable of recognizing the class in which the device is working and in consequence give a reasonable output response.
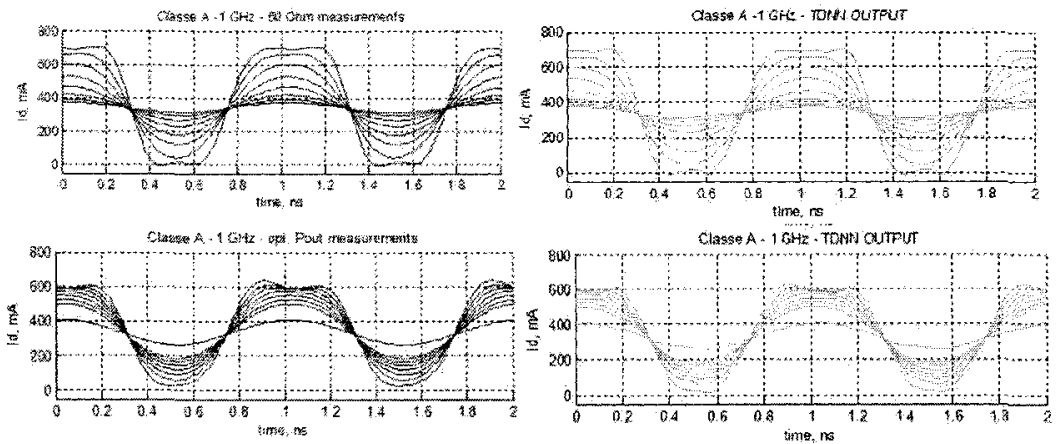

Fig. 8. Time domain waveforms at $1 \mathrm{GHz}$ at increasing power for class $\mathrm{A}$ at $50 \mathrm{Ohm}$ (used for training), and optimum Pout (used for validation). Measurements (left), TDNN output (right).

\section{Conclusions}

In this paper, a model that has the capability of learning and predicting the dynamic behavior of nonlinear PAs, based on a Time-Delayed Neural Network (TDNN), has been proposed. Validation and accuracy of the TDNN model in the time-domain showed good agreements between the TDNN model output data and measurements.

The TDNN model can be trained with input/output device measurements or simulations, and a very good accuracy can be obtained in the device characterization easily and rapidly. These properties make this kind of models specially suitable for new wireless communications components modeling, which are mostly nonlinear and require speed, accuracy and simplicity when designing and building the model.

\section{Acknowledgement}

The authors would like to thank Prof. Marco Pirola and Vittorio Camarchia, from Politecnico di Torino at Turin (IT), for providing the measurements used in this work. Also, we would like to thank Banco Ro and Red Universia for the financial support under project number INV-1334. 


\section{References}

1. Evci C, Barth U, Sehier P, Sigle R. (2003) The path to beyond 3G systems: strategic and technological challenges. In: Proc. $4^{\text {th }}$ Int. Conf. on $3 \mathrm{G}$ Mobile Communication Technologies, pp. 299-303

2. Elwan H, Alzaher H, Ismail M (2001) New generation of global wireless compatibility. IEEE Circuits and Devices Magazine 17: 7-19

3. Ahmed A, Abdalla MO, Mengistu ES, Kompa G. (2004) Power Amplifier Modeling Using Memory Polynomial with Non-uniform Delay Taps. In: Proc. IEEE $34^{t h}$ European Microwave Conf., pp. 1457-1460

4. Ku H, Kenney JS (2003) Behavioral Modeling of Nonlinear RF Power Ampli-fiers considering memory effects. IEEE Trans. Microwave Theory Tech. 51: 2495-2504

5. Root D, Wood J (2005) Fundamentals of Nonlinear Behavioral Modeling for RF and Microwave design. Artech House, Boston

6. Zhang QJ, Gupta KC, Devabhaktuni VK (2003) Artificial Neural Networks for $\mathrm{RF}$ and Microwave Design - From Theory to practice. IEEE Trans. Microwave Theory Tech. 51: 1339-1350

7. Zhang QJ, Gupta KC (2000) Neural Networks for RF and Microwave Design. Artech House, Boston

8. Schreurs D, Verspecht J, Vandamme E, Vellas N, Gaquiere C, Germain M, Borghs G (2003) ANN model for AlGaN/GaN HEMTs constructed from near-optimalload large-signal measurements. IEEE Trans. Microwave Theory Tech. 51: 447450

9. Liu T, Boumaiza S, Ghannouchi FM (2004) Dynamic Behavioral Modeling of 3G Power Amplifiers Using Real-Valued Time-Delay Neural Networks. IEEE Trans. Microwave Theory Tech. 52: 1025-1033

10. Ahmed A, Srinidhi ER, Kompa G (2005) Efficient PA modelling using Neural Network and Measurement setup for memory effect characterization in the power device. In: Proc. IEEE MTT-S International Microwave Symposium, pp. 18711874

11. Alabadelah A, Fernandez T, Mediavilla A, Nauwelaers B, Santarelli A, Schreurs D, Tazón A, Traverso PA (2004) Nonlinear Models of Microwave Power Devices and Circuits. In: Proc. $12^{\text {th }}$ GAAS Symposium, pp. 191-194

12. Xu J, Yagoub MCE, Ding R, Zhang QJ (2002) Neural-based dynamic modeling of nonlinear microwave circuits. IEEE MTT-S Int. Microwave Symp. Dig. 1: 11011104

13. Liu T, Boumaiza S, Ghannouchi FM (2004) Dynamic Behavioral Modeling of 3G Power Amplifiers using real-valued Time-Delay Neural Networks. IEEE Trans. Microwave Theory Tech. 52: 1025-1033

14. Nguyen D, Widrow B (1990) Improving the learning speed of 2-layer neural networks by choosing initial values of the adaptive weights. In: Proc. IEEE Int. Joint Conf. Neural Networks, vol. 3, pp. 21-26

15. Marquardt DW (1963) An algorithm for least-squares estimation of non-linear parameters. Journal of the Society for Industrial and Applied Mathematics 11: 431-441

16. Sjoberg J, Ljung L (1995) Overtraining, regularization and searching for a minimum, with application to neural networks. Int. J. Control 62: 1391-1407 